\section{Costos de un programa de control del vector Aedes aegypti en municipios de Colombia: el caso de Girón y Guadalajara de Buga, 2016}

\author{
Costs of an Aedes aegypti vector control program \\ in municipalities in Colombia: a case study in \\ Girón and Guadalajara de Buga, 2016
}

\section{Custos de um programa de controle ao vetor Aedes aegypti em municípios da Colômbia: o caso de Girón e Guadalajara de Buga, 2016}

Manuel Alejandro Salinas-López 1 Victoria Eugenia Soto-Rojas 1,2 Clara Beatriz Ocampo 1,3

doi: 10.1590/0102-311X00044518

\section{Resumen}

El objetivo de este estudio fue cuantificar los costos de los programas de control de enfermedades transmitidas por vectores a nivel local en Colombia (2016). Para ello, fue realizado un análisis de costos desde la perspectiva del hacedor de politica en los municipios de Girón y Guadalajara de Buga, específicamente para el programa de control del Aedes aegypti, principal vector trasmisor del dengue, Zika y chikungunya. Este análisis implicó la cuantificación de todos los costos requeridos para cada una de las estrategias de prevención y control de enfermedades transmitidas por vectores. Los costos fueron clasificados en recurrentes y de capital, además, con fines comparativos se obtuvo el costo por caso y a nivel per cápita. El costo total estimado de los programas fue de USD 146.651 en el municipio de Girón y USD 97.936 en el municipio de Guadalajara de Buga; a nivel per cápita el costo fue de USD 0,88 en Girón y en Guadalajara de Buga fue de USD 0,99. En general, las estrategias predominantes en costos fueron el control químico de vectores adultos, con un $26 \%$ del total de los costos en Girón y un 47\% en Guadalajara de Buga, esta fue intensiva en costos recurrentes, con un costo de personal $40 \%$ del total de costos para esta estrategia en Girón y un 66\% de costos operacionales en Guadalajara de Buga.

Dengue; Costos y Análisis de Costo; Control de Vectores

\author{
Correspondencia \\ M. A. Salinas-López \\ Universidad Icesi. \\ Calle 18, № 122-135, Oficina - Casa Rocha 2o Piso, Cali, Valle \\ del Cauca - 760031, Colombia. \\ manuelsalis@hotmail.com \\ 1 Universidad Icesi, Cali, Colombia. \\ 2 Centro de Estudios en Protección Social y Economía de la \\ Salud, Cali, Colombia. \\ 3 Centro Internacional de Entrenamiento e Investigaciones \\ Médicas, Cali, Colombia.
}




\section{Introducción}

El Aedes (stegomyia) aegypti, y en algunos casos Aedes albopictus, son los vectores responsables de la transmisión en zonas urbanas de los virus de dengue, Zika y chikungunya, entre otros. Estas enfermedades infecciosas representan una carga importante en términos de morbilidad para el mundo y principalmente en los países del trópico. A nivel mundial, se estima que el virus del dengue afecta a 390 millones de personas al año ${ }^{1}$ y más de 2,5 billones de personas están en situación de riesgo en alrededor de 128 países 2. En Colombia, se estima que el dengue afectó a cerca de 103 mil personas en el 2016, y cerca de 106 mil personas fueron infectadas con Zika, entre el año 2015 y 2016 3. El dengue se ha caracterizado por presentar un comportamiento cíclico de epidemias que se repite de cada tres a cuatro años 4 . Cerca del 55\% de la población colombiana se encuentra en riesgo de contraer la enfermedad o morir a causa de ella 5 .

En términos económicos el dengue representa un costo para el mundo que va de los USD 8 a 9 billones al año, lo que implica un costo promedio anual por caso de USD 1516 . Para Colombia, las estimaciones del costo de la enfermedad indican un costo total de USD 167 millones en el año 2010, con un registro de 292 muertes confirmadas por dengue para ese año 7, representando el 4,73\% de las defunciones por enfermedades infecciosas y parasitarias 8 , siendo el segundo país con mayores defunciones por dengue para ese año, precedido por Brasil con un registro de 656 muertes (Organización Mundial de la Salud. http://www.paho.org/data/index.php/es/temas/indicadores-dengue/ dengue-nacional/238-dengue-mortalidad-tasa.html, accedido el 01/Feb/2018). Desde una perspectiva global del costo de la enfermedad, el dengue ha representado una carga para el mundo de 1 a 14 millones DALYs (disability adjusted life years) en el 2013 con un promedio de 9.221 casos fatales al año ${ }^{9}$. En Colombia la carga para la sociedad de esta enfermedad se estimó en 57.017 DALY para el brote del año 20107 .

En Colombia, las estrategias de control vectorial son ejecutadas y planeadas por entes territoriales descentralizados. De acuerdo a indicadores, como la población e ingresos corrientes de libre destinación de los entes territoriales, las estrategias pueden ser ejecutadas y planeadas por los municipios o departamentos. En los municipios de mayor categoría, las competencias de control de las enfermedades transmitidas por vectores (ETV) recaen sobre ellos, mientras que para los de menor categoría la responsabilidad recae sobre el departamento 5. Los municipios ejecutan y planean las estrategias del control del vector, siguiendo las guías del Instituto Nacional de Salud (INS) Colombiano, que a su vez se basa en los lineamientos dados por la Organización Mundial de la Salud y la Organización Panamericana de la Salud 10.

Pese a la importancia de estas iniciativas gubernamentales, especialmente paras las regiones con menos recursos; no existe documentación -al menos de nuestro conocimiento- del costo promedio de los programas municipales de control y prevención del dengue en Colombia, los estudios existentes se han enfocado sobre todo en la estimación de la carga de la enfermedad 7, en la estimación de los costos para el sistema de salud y el gasto de bolsillo de los pacientes 11 . Sin embargo, a nivel internacional se observa un interés en documentar el costo de los programas y rutinas de control y prevención del dengue a nivel nacional y local 12,13,14,15, como insumo necesario para el planeamiento e implementación de programas de ETV a nivel local, particularmente con presupuestos fijos.

Dada la escasa documentación, en relación con los costos de un programa municipal de control y prevención del dengue en Colombia, este estudio tiene como objetivo analizar los costos de los programas de control y prevención del dengue, ejecutados en el año 2016, para dos municipios. Para ello, se analiza la estructura de los costos de los programas, se cuantifica los costos por estrategias ejecutadas y analiza la tendencia mensual de estos, contrastada con el comportamiento de la incidencia mensual del dengue para cada municipio. Este estudio no sólo permitirá costear las acciones de ETV a nivel municipal en Colombia, sino que también es útil para la gestión de los programas a nivel municipal. Cuantificar los programas locales es importante, dado que los recursos con los que cuentan los municipios son escasos y es importante focalizar y dirigir los recursos de manera eficiente, además de servir como un insumo para posteriores estudios a nivel de costo-efectividad. 


\section{Métodos}

\section{Área de estudio}

Se seleccionaron los municipios de Girón en el departamento de Santander y Guadalajara de Buga en el Valle del Cauca, municipios participantes dentro del proyecto Desarrollo, Implementación y Evaluación de un Sistema Prototipo que Integra Vigilancia, Diseño de Estrategias de Prevención e Intervención y Evaluación de Impacto para el Control de Dengue, proyecto 1 del programa Investigación Aplicada para Contribuir a un Modelo Efectivo y Sostenible de Intervención del Dengue en Santander, Casanare y Valle del Cauca. Este programa fue financiado por el Sistema General de Regalías de CTeI (Acuerdo 009, 23 agosto, 2013). Los entes territoriales estudiados fueron escogidos por el carácter endémico del dengue y por tener un programa de control de vectores autónomo.

Girón es un municipio ubicado en el departamento de Santander, en la región centro-oriental del país, forma parte del área metropolitana de Bucaramanga, capital del departamento; cuenta con una población proyectada de 190.350 habitantes, de los cuales el 90\% se ubica en las zonas de cabecera 16 . Los virus transmitidos por el principal vector $A$. aegypti son un problema de salud pública para este municipio. En los últimos tres años se reportaron incidencias anuales de dengue de 812, 450 y 540 casos por cien mil habitantes en los años 2014, 2015 y 2016 respectivamente 3, esta enfermedad ha sido considerada como endémica del municipio.

El municipio de Guadalajara de Buga se encuentra ubicado en el departamento del Valle del Cauca en la región sur-occidental del país, cuenta con una población aproximada de 114.798 habitantes, la cual es predominantemente urbana, con una concentración del $86 \%$ de la población en cabeceras urbanas 17 . En cuanto a la situación del dengue en este municipio, en los últimos tres años se reportaron incidencias anuales de dengue de 508, 293 y 560 por 100 mil habitantes en los años 2014, 2015 y 20163.

\section{Programa de control de vectores}

Los dos municipios analizados presentan diferencias en la disposición institucional de la implementación de su programa de ETV. El municipio de Girón tiene una dependencia destinada exclusivamente a las ETV. La coordinación de ETV tiene un personal a cargo, disponible la mayor parte del año, y el equipo se encarga de ejecutar directamente todas las actividades de control. En el municipio de Guadalajara de Buga el programa de ETV es manejado por la dependencia de Saneamiento Ambiental, que además se encarga de actividades de salud ambiental, control de alimentos, plagas, zoonosis, potabilidad de aguas y sanidad. En términos de gestión, el programa de Guadalajara de Buga es ejecutado por contratación externa de las actividades de ETV. Ambos municipios ejecutan estrategias similares, el Cuadro 1 resume las actividades ejecutadas en el año 2016 en ambos municipios, los encargados del control de las ETV combinan un conjunto de estrategias que incluyen el control químico de vectores, la vigilancia entomológica, trabajo con la comunidad y alteración del medio que propicia la propagación del vector.

\section{Recolección de datos}

Los datos corresponden a los recursos incurridos y ejecutados por la coordinación de ETV de Girón y la coordinación de saneamiento del municipio de Guadalajara de Buga en el año 2016. Los costos fueron recolectados empleando la metodología de micro costeo de abajo hacia arriba, cuantificando los recursos empleados en cada una de las actividades ejecutadas mensualmente ${ }^{12}$. Los costos fueron clasificados por actividad y en costos recurrentes y de capital, de acuerdo con la metodología de la OMS 13. Se adjudicaron los costos a cada una de las actividades detalladas en el Cuadro 1 , y en la clasificación de costos recurrentes se incluyeron los costos del personal empleado, materiales e insumos (insecticidas, larvicidas, materiales de oficina, elementos de protección), costos de transporte (gasolina, alquiler de vehículo, aceite), difusión (volantes, pauta en radio y folletos), mantenimiento (mantenimiento de equipos de fumigación, equipos de oficina, equipos de comunicación), servicios contratados (en Guadalajara de Buga las actividades de ETV son subcontratadas) e indirectos (un 12\% 


\section{Cuadro 1}

Actividades del programa de enfermedades trasmitidas por vectores en los municipios de Girón y Guadalajara de Buga, Colombia, 2016.

\begin{tabular}{|l|c|c|}
\hline \multirow{2}{*}{ Estrategia } & GIRÓN & GUADALAJARA DE BUGA \\
\cline { 2 - 3 } & Actividades & Actividades \\
\hline Control químico & Aplicación de larvicidas & Aplicación de larvicidas \\
& Aplicación de insecticidas & Contrón de insecticidas \\
& Control de sumideros & Capacitación de personas \\
\hline Movilización y comunicación social & Capacitación de la comunidad & \\
& Entrega de toldillos TILD & \\
& Campañas de educación & \\
\hline Vigilancia entomológica & Visita a los casos reportados & Levantamiento entomológico \\
\hline Alteración del medio ambiente & Levantamiento entomológico & Fumigación ambiental \\
\hline
\end{tabular}

TILD: toldillos con insecticida de larga duración.

Fuente: elaboración propia basada en datos recolectados en campo.

de los costos recurrentes, incluye jurídicos, mensajería, apoyo administrativo de otras dependencias de la alcaldía, uso de instalaciones).

La clasificación de costos de capital incluye todos los costos asociados al uso y adquisición de equipos duraderos, por ejemplo, equipos de fumigación, equipos de oficina, equipos de comunicación y un software de gestión integrada y referenciación geográfica de los casos de ETV, llamado Vectos, desarrollado por el proyecto para los municipios participantes. El planeamiento de las estrategias realizadas por los municipios fue tomado del registro de actividades trimestrales presupuestadas, metas de indicadores en salud y actividades ejecutadas, conocido como el POA (Plan Operativo Anual).

La información relacionada con los costos fue tomada de la contabilidad del municipio, de los presupuestos realizados por los coordinadores de ETV, y de la bitácora diaria de actividades realizadas e insumos empleados en cada actividad. Se verificó información con el registro documental de contratos, esta información fue suministrada por los municipios, así como por el personal de CIDEIM (Centro Internacional de Entrenamiento e Investigaciones Médicas). Los costos fueron recolectados en pesos colombianos (COP) del año 2016 y se convirtieron a dólares americanos (USD), con la tasa de cambio de USD 1 = COP 3.053,42. Esta tasa de cambio corresponde al promedio anual de la tasa representativa del mercado en el 2016.

Los casos de dengue reportados en los municipios estudiados fueron tomados del Sistema Nacional de Vigilancia en Salud Pública de Colombia (SIVIGILA). Estos casos corresponden a los reportados hasta la semana 52 del 2016 en cada municipio e incluyen tanto casos confirmados por laboratorio, así como sospechosos.

\section{Análisis de la información}

Los costos fueron estimados anualmente, por actividad, por caso y por habitante. Asimismo, se discriminaron los costos de las actividades ejecutadas por costos de personal, operacionales, insumos y capital. Los costos de insumos administrativos, los costos de transporte, costos de mantenimiento e indirectos fueron agrupados como costos operacionales. En la clasificación de los costos se diferencia entre recurrentes y de capital, los costos recurrentes se refieren a aquellos costos incurridos periódicamente, y cuya durabilidad de uso es menor a un año o es inmediata, mientras que los costos de capital se refieren al costo de adquirir bienes, que tiene una vida útil mayor a un año y cuya finalidad es ser parte del proceso de elaboración de un bien final. Los costos de capital fueron depreciados de 
forma lineal, teniendo en cuenta la vida útil y los años de uso, así mismo los costos de capital fueron valorados con una tasa de descuento del $3 \%$, equivalente a la inflación de largo plazo en Colombia.

Con el fin de realizar un contraste de costos incurridos y resultados en salud, se calculó la incidencia del dengue por cien mil habitantes. Esta incidencia fue calculada como la razón entre los casos reportados de dengue en el 2016 sobre la población de cabecera urbana, proyectada por el Departamento Administrativo Nacional de Estadística (DANE) para el 2016, multiplicada por 100 mil. Se tomó sólo la población urbana, debido al carácter urbano de la trasmisión de esta arbovirosis 14. Para el procesamiento de los datos se empleó el software Microsoft Excel 2013 (https://products.office.com/).

\section{Resultados}

El costo total del programa de control de vectores en el municipio de Girón fue de USD 146.651 para el 2016, las actividades más costosas fueron el uso del sistema de gestión integrado Vectos (USD 40.770, 28\% del total de costos), seguido por el control químico de vectores adultos (USD 37.643, 25\% del total de costos), y la capacitación y difusión de información en la comunidad (USD 20.850, 14\% del total de los costos) (Tabla 1). En cuanto a las medidas de costo individual, se estimó que el costo anual del programa de control de vectores de Girón por caso reportado fue de USD 327 y per cápita fue de USD 0,88 (Tabla 1).

Las estrategias de ETV, a excepción del uso del sistema Vectos y la entrega de toldillos TILD (toldillos con insecticida de larga duración de la marca PermaNet, Vestergaard Frandsen Inc., Lausana, Suiza) fueron intensivas en el uso de personal, este rubro representó cerca del 58\% de los costos de la estrategia de control de sumideros y del vector en estadio larvario. Un $40 \%$ en el control químico del vector adulto y las campañas de capacitación; mientras que para las demás actividades como: la visita de los casos reportados, la recolección de recipientes inservibles, los levantamientos entomológicos y la actualización de la línea base de ETV, el personal representó entre el 74\% y el 89\% de los costos de cada actividad (Tabla 1).

El uso del capital en las estrategias implementadas en Girón fue intensivo en estrategias como el uso del sistema integrado de gestión Vectos y la entrega de toldillos TILD. Dado que Vectos es un software, se catalogó como costo de capital su implementación y uso, así como todos los elementos y material intelectual empleado en la creación del sistema. Mientras que los toldillos TILD, fueron catalogados como un bien de capital, dado que la literatura ha determinado que estos tienen una vida útil efectiva de tres años 15 . El capital representa para la estrategia Vectos el 100\% de los costos, mientras que para la entrega de toldillos TILD representa un 54\% del total de los costos de la estrategia. Dado que el programa de control de vectores de Girón es contratado directamente por el municipio, otras estrategias como el control químico de vectores adultos tiene un importante uso de capital (24\%) (Tabla 1).

Los insumos como los insecticidas representaron un $16 \%$ de los costos de las estrategias de control químico de vectores adultos (Tabla 1). El programa de control vectorial de Girón utilizó aproximadamente 113 litros de malathion y 130 litros de Pirilan (Syngenta Crop Protection, Bogotá, Colombia) en la fumigación en el interior y exterior de las viviendas. Mientras que en las actividades de control larvario: los larvicidas representaron un 12\% del total de los costos (Tabla 1). En control de sumideros en las calles y criaderos en viviendas se empleó $36 \mathrm{~kg}$ de temefos, 96 unidades de biolarvicidas y 146 pastillas de Dimilin (Chemtura Corp., EE.UU.).

Por otra parte, la Figura 1 muestra la ejecución mensual de estos costos, y la incidencia mensual de casos reportados de dengue en el 2016 para el municipio de Girón, donde se puede destacar una mayor ejecución de recursos en el primer semestre del año, lo cual coincide con el mayor número de casos reportados. En el primer semestre, se observa que el mes de marzo presenta el mayor costo, aproximadamente USD 19.000 ejecutados, y el menor monto ejecutado corresponde al mes de enero con un poco más de USD 2.000. Esta situación se presenta debido a que la contratación de personal tarda cerca de dos meses, lo que provoca que enero y febrero el número de actividades ejecutadas sea menor. De igual manera, el mayor pico de incidencia en el 2016 se presentó en el mes de mayo con cerca de 45 casos por 100 mil habitantes. 
Tabla 1

Costo anual, por caso y per cápita de las estrategias de enfermedades trasmitidas por vectores (ETV) en el municipio de Girón, Colombia.

\begin{tabular}{|c|c|c|c|c|c|}
\hline Estrategias/Recursos & $\begin{array}{l}\text { Costo anual } \\
\text { (USD) }\end{array}$ & $\begin{array}{c}\text { Costo por caso } \\
\text { (USD) }\end{array}$ & $\begin{array}{c}\text { Costo per cápita } \\
\text { (USD) }\end{array}$ & $\begin{array}{c}\text { Subtotal } \\
(\%)\end{array}$ & $\begin{array}{c}\text { Total } \\
(\%)\end{array}$ \\
\hline Sumideros y control larvario & 8.560 & 19,06 & 0,05 & 100 & 6 \\
\hline Personal & 4.943 & 11,01 & 0,03 & 58 & 3 \\
\hline Operacionales & 1.410 & 3,14 & 0,01 & 17 & 1 \\
\hline Larvicidas & 1.055 & 2,35 & 0,01 & 12 & 1 \\
\hline Capital & 1.152 & 2,56 & 0,01 & 13 & 1 \\
\hline Control químico de vectores adultos & 37.643 & 83,84 & 0,23 & 100 & 25 \\
\hline Personal & 15.119 & 33,67 & 0,09 & 40 & 10 \\
\hline Operacionales & 5.744 & 12,79 & 0,03 & 16 & 4 \\
\hline Insecticidas & 6.178 & 13,76 & 0,04 & 16 & 4 \\
\hline Capital & 10.602 & 23,61 & 0,06 & 28 & 7 \\
\hline Capacitación y difusión & 20.850 & 46,44 & 0,13 & 100 & 14 \\
\hline Personal & 8.379 & 18,66 & 0,05 & 40 & 6 \\
\hline Operacionales & 7.555 & 16,83 & 0,05 & 36 & 5 \\
\hline Capital & 4.915 & 10,95 & 0,03 & 24 & 3 \\
\hline Visitas a los casos SIVIGILA & 5.199 & 11,58 & 0,03 & 100 & 4 \\
\hline Personal & 4.190 & 9,33 & 0,03 & 81 & 3 \\
\hline Operacionales & 1.009 & 2,25 & 0,01 & 19 & 1 \\
\hline Recolección de inservibles & 4.928 & 10,97 & 0,03 & 100 & 3 \\
\hline Personal & 4.190 & 9,33 & 0,03 & 85 & 2 \\
\hline Operacionales & 738 & 1,64 & 0,00 & 15 & 1 \\
\hline Levantamiento entomológico & 6.659 & 14,83 & 0,04 & 100 & 5 \\
\hline Personal & 4.943 & 11,01 & 0,03 & 74 & 3 \\
\hline Operacionales & 1.299 & 2,89 & 0,01 & 20 & 2 \\
\hline Capital & 417 & 0,93 & 0,00 & 6 & 0 \\
\hline Entrega de toldillos TILD & 17.185 & 38,27 & 0,10 & 100 & 12 \\
\hline Personal & 4.190 & 9,33 & 0,03 & 24 & 3 \\
\hline Operacionales & 3.687 & 8,21 & 0,02 & 22 & 3 \\
\hline Capital & 9.308 & 20,73 & 0,06 & 54 & 6 \\
\hline Sistema de gestión integrado (Vectos) & 40.770 & 90,80 & 0,25 & 100 & 28 \\
\hline Capital & 40.770 & 90,80 & 0,25 & 100 & 28 \\
\hline Actualización de la línea base de ETV & 4.859 & 10,82 & 0,03 & 100 & 3 \\
\hline Personal & 4.301 & 9,58 & 0,03 & 89 & 3 \\
\hline Operacionales & 558 & 1,24 & 0,00 & 11 & 0 \\
\hline Total & 146.651 & 326,62 & 0,88 & & 100 \\
\hline
\end{tabular}

SIVIGILA: Sistema Nacional de Vigilancia en Salud Pública de Colombia; TILD: toldillos con insecticida de larga duración.

Fuente: elaboración própria.

Por otro lado, se tiene al municipio de Guadalajara de Buga, donde el costo total del programa de control de vectores para el 2016 fue de USD 97.936. Las actividades más costosas fueron el control químico de vectores adultos (USD 46.155, 47\% del total de costos) y el control larvario en criaderos y sumideros (USD 37.072, 38\% del total de costos) (Tabla 2). El costo total por caso reportado fue de USD 177, inferior al costo por caso de Girón (USD 326) y el costo per cápita fue de USD 0,99, levemente superior al costo per cápita de Girón (USD 0,88) (Tabla 2).

El conjunto de estrategias del programa de vectores de Guadalajara de Buga resultó ser intensivo en costos corrientes, más del $95 \%$ de los costos se distribuye en los rubros de costes de personal, operacionales e insumos de larvicidas e insecticidas. El mayor componente de los costos de las estrategias resultó ser los costes operacionales, un $66 \%$ de los costos de la estrategia de control químico 


\section{Figura 1}

Costos mensuales e incidencia mensual del dengue por 100 mil habitantes. Girón, Colombia, 2016.

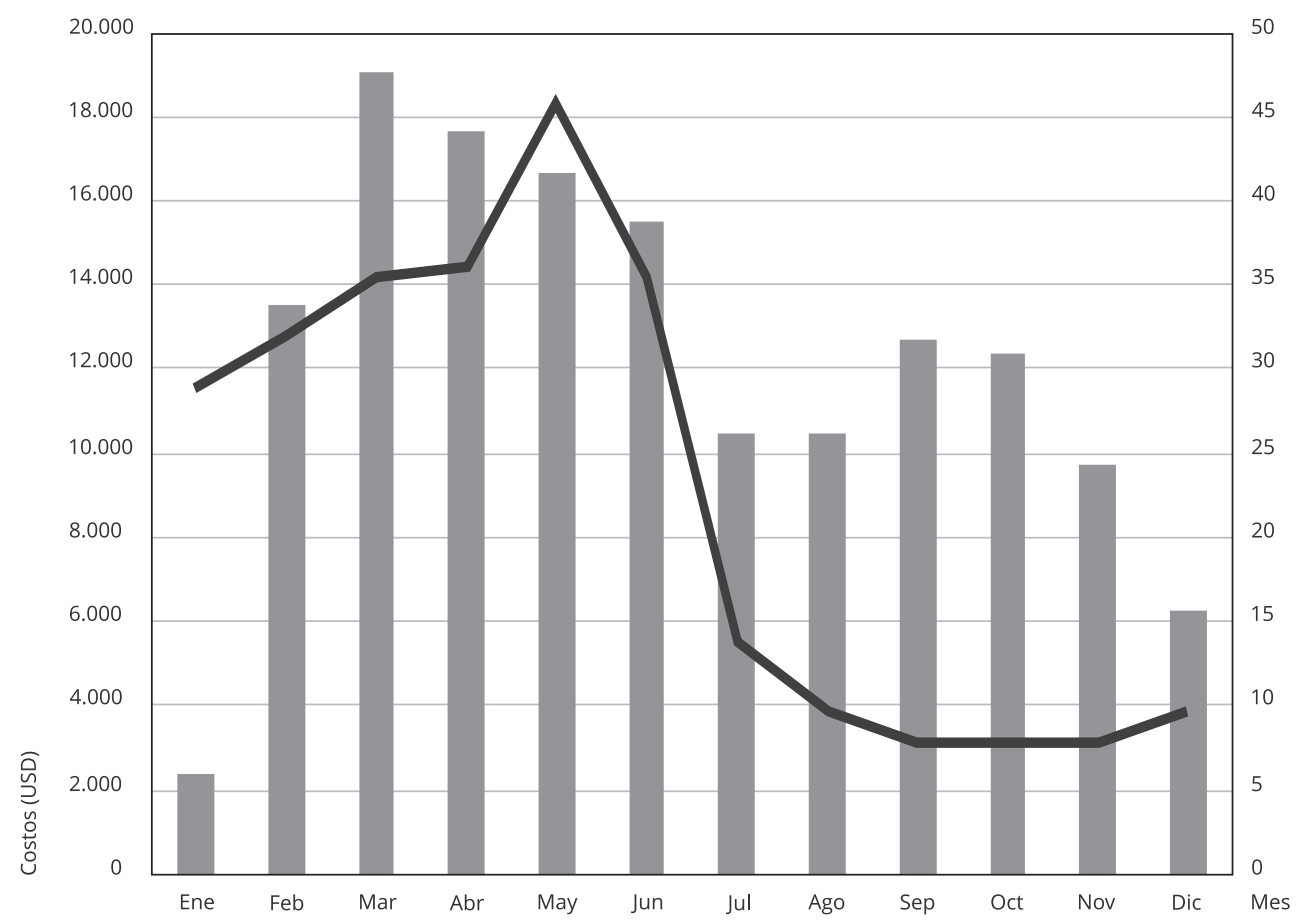

Costos mensuales

- Incidencia mensua del dengue

de vectores adultos, un $49 \%$ de los costos de control larvario en sumideros y criaderos, un $77 \%$ en los costos de capacitación, un 60\% de los costos de los levantamientos entomológicos y un 55\% de los costos de fumigación ambiental (Tabla 2). Esta distribución de los costos se debió a que el programa de control de vectores de Guadalajara de Buga subcontrata la implementación de las estrategias, es decir, la coordinación de ETV y salud ambiental de Guadalajara de Buga planea las actividades, pero la ejecución de estas es realizada por terceros.

Dado que la ejecución de las estrategias es realizada por terceros, los costos de capital resultan ser inferiores, ya que los bienes de capital son propiedad del tercero que ejecuta las estrategias de ETV, es decir, el municipio paga un tipo de arriendo por el uso de los equipos y maquinaria. Los costos de capital, registrados en la Tabla 2, corresponden a los equipos de oficina, tales como computadores, sillas, teléfonos, impresoras, mesas, entre otros. Los costos de estos bienes fueron estimados a partir de los precios internacionales disponibles en el sitio web WHO-CHOICE (http://www.who.int/choice/ cost-effectiveness/inputs/capital_goods/en/, accedido el 25/May/2017). El valor de los bienes de capital fue traído a precios del 2016, como supuesto se le asignó el costo a las actividades de ETV de manera igual, pero el valor de estos bienes de capital fue distribuido de acuerdo a la intensidad de uso dado, que son usados por otras actividades que no son ETV, como saneamiento, por las características de la dependencia en Guadalajara de Buga.

En cuanto a los resultados del programa de ETV de Guadalajara de Buga, la Figura 2 muestra la ejecución mensual de los costos del programa de ETV y la incidencia mensual de casos reportados de dengue en el 2016 para el municipio de Guadalajara de Buga. De esta gráfica se puede destacar que, a diferencia del municipio de Girón, Guadalajara de Buga presenta su mayor pico de incidencia en el mes de febrero con cerca de 120 casos por 100 mil habitantes, es decir, tres meses antes que Girón y a una escala superior (Girón: mayo y 45 casos por 100 mil habitantes). 
Tabla 2

Costo anual, por caso y per cápita de las estrategias de enfermedades trasmitidas por vectores en el municipio de Guadalajara de Buga, Colombia.

\begin{tabular}{|c|c|c|c|c|c|}
\hline Actividades/Recursos & $\begin{array}{l}\text { Costo anual } \\
\text { (USD) }\end{array}$ & $\begin{array}{c}\text { Costo por caso } \\
\text { (USD) }\end{array}$ & $\begin{array}{c}\text { Costo per cápita } \\
\text { (USD) }\end{array}$ & $\begin{array}{c}\text { Subtotal } \\
(\%)\end{array}$ & $\begin{array}{c}\text { Total } \\
\text { (\%) }\end{array}$ \\
\hline Sumideros y control larvario & 37.072 & 66,92 & 0,37 & 100 & 38 \\
\hline Personal & 14.362 & 25,92 & 0,15 & 39 & 15 \\
\hline Operacionales & 18.254 & 32,95 & 0,18 & 49 & 19 \\
\hline Insecticida y larvicidas & 4.270 & 7,71 & 0,04 & 12 & 4 \\
\hline Capital & 185 & 0,33 & 0,00 & 0 & 0 \\
\hline Control químico de vectores adultos & 46.155 & 83,31 & 0,47 & 100 & 47 \\
\hline Personal & 14.187 & 25,61 & 0,14 & 31 & 14 \\
\hline Operacionales & 30.512 & 55,08 & 0,32 & 66 & 31 \\
\hline Insecticidas & 1.271 & 2,29 & 0,01 & 3 & 2 \\
\hline Capital & 185 & 0,33 & 0,00 & 0 & 0 \\
\hline Capacitación y difusión & 3.576 & 6,46 & 0,04 & 100 & 4 \\
\hline Personal & 639 & 1,15 & 0,01 & 18 & 1 \\
\hline Operacionales & 2.752 & 4,97 & 0,03 & 77 & 3 \\
\hline Capital & 185 & 0,33 & 0,00 & 5 & 0 \\
\hline Levantamiento entomológico & 4.281 & 7,73 & 0,04 & 100 & 4 \\
\hline Personal & 1.508 & 2,72 & 0,02 & 35 & 2 \\
\hline Operacionales & 2.588 & 4,67 & 0,03 & 60 & 2 \\
\hline Capital & 185 & 0,33 & 0,00 & 5 & 0 \\
\hline Fumigación ambiental & 6.852 & 12,36 & 0,07 & 100 & 7 \\
\hline Personal & 1.289 & 2,33 & 0,01 & 19 & 1 \\
\hline Operacionales & 3.819 & 6,89 & 0,04 & 55 & 4 \\
\hline Insecticidas & 1.558 & 2,81 & 0,02 & 23 & 2 \\
\hline Capital & 185 & 0,33 & 0,00 & 3 & 0 \\
\hline Total & 97.936 & 176,78 & 0,99 & & 100 \\
\hline
\end{tabular}

Fuente: elaboración propia.

La reducción de la incidencia mensual en Guadalajara de Buga coincide con una mayor ejecución de recursos durante el periodo, comprendida entre febrero y junio, alcanzando el mayor pico de recursos ejecutados en mayo con cerca de USD 15.000 mensuales. Durante el segundo semestre se presentan las incidencias más bajas (por debajo de los 20 casos por 100 mil habitantes) y estas coinciden con una mayor ejecución de recursos en el programa de ETV. Sin embargo, también se presentan dos periodos muertos de baja ejecución de recursos en julio y agosto. Al igual que en Girón, Guadalajara de Buga presenta un tiempo de demora en la contratación de los elementos que requiere para ejecutar el programa de ETV, sólo que este lapso de tiempo es mayor y se extiende hasta el mes de abril (Figura 2).

\section{Discusión}

Los resultados hallados para los municipios de Girón y Guadalajara de Buga muestran dos municipios de similares características, con programas de ETV con costos y estrategias diferentes. Girón presenta un programa de control vectorial más diversificado, en cuanto a estrategias, que el municipio de Guadalajara de Buga, mientras que Girón ejecuta cerca de 9 estrategias, el municipio de Guadalajara de Buga ejecuta 6 estrategias. Esta diversificación de las estrategias se vio reflejada en la distribución de los costos entre estrategias. Por ejemplo, los recursos del programa de ETV de Guadalajara de Buga estuvieron enfocados en estrategias de control del vector adulto, con un $47 \%$ de los costos y el control 


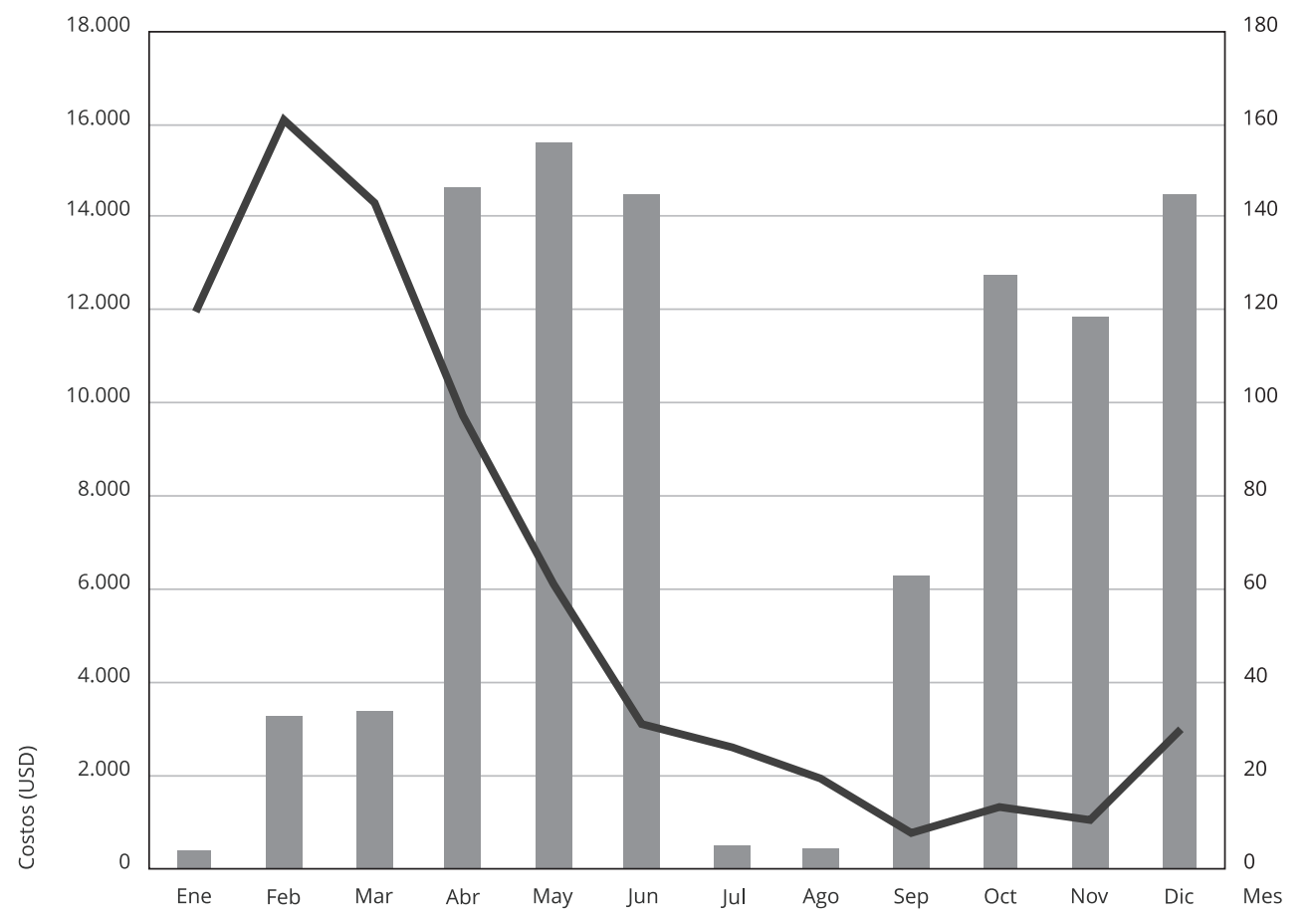

Costos mensuales

- Incidencia mensua del dengue

larvario con 38\%, mientras que el programa del municipio de Girón para estas mismas estrategias tiene un $25 \%$ y un $6 \%$ del total de los costos respectivamente, ganando peso en las estrategias comunitarias, como capacitación de la comunidad con un 14\%, y la entrega de toldillos TILD con un $12 \%$. Este resultado es previsible, dado que en Colombia los lineamientos proporcionados por el gobierno nacional son una guía, la cual los municipios tienen libertad de adoptar o no.

En cuanto a la planificación y ejecución de estrategias, se evidencia diferencias notables entre los dos programas. Por un lado, tenemos que el municipio de Guadalajara de Buga no tiene una dependencia dedicada exclusivamente a asuntos de ETV, sino que la responsabilidad recae sobre la dependencia de sanidad ambiental. Por otra parte, la ejecución de las estrategias es realizada por terceros, bajo un esquema de subcontratación de las actividades de ETV. Esta situación coincide con un lapso prolongado, en que no se ejecutan recursos en actividades de ETV (desde el mes de enero al mes de abril), periodo en el que además se presenta el mayor brote de incidencia de dengue del año 2016, con cerca de 120 casos por 100 mil habitantes. Mientras que el municipio de Girón posee una dependencia dedicada exclusivamente a ETV, con un personal de planta y bienes de capital propios, que pueden dar una mayor capacidad de reacción ante eventuales brotes de la enfermedad. Por ejemplo, el lapso de contratación en el que no se ejecutan actividades se extiende sólo hasta el mes de febrero, a partir de ese mes se logran ejecutar recursos que desplazan el brote al mes de mayo a un nivel inferior al de Guadalajara de Buga con cerca de 45 casos por 100 mil habitantes.

Frente a lo expuesto en el párrafo anterior, si se contrasta un indicador construido por el Departamento Nacional de Planeación de eficacia municipal, entendida como el nivel de cumplimiento de los municipios de las metas definidas en el Plan de Desarrollo municipal 18, se encuentra que Girón mostró un mejor indicador de gestión de su planeamiento, al reportar un indicador de eficacia en el 2015 del 100\% 16, frente al 46\% que reportó el municipio de Guadalajara de Buga para el mismo año, 
aunque presentó una leve mejora frente al 2012, cuando reportó un indicador de eficacia del 36\% 17. En general, el indicador de desempeño integral que integra los componentes de eficacia, eficiencia, ejecución de recursos y gestión administrativa fue superior en Girón con un 90\% en el 2015 16, frente un $75 \%$ del municipio de Guadalajara de Buga para el mismo año ${ }^{17}$. Estos indicadores señalan que en el municipio hay un mejor desempeño de la planeación y ejecución relativo al municipio de Guadalajara de Buga.

En cuanto a la composición de los costos de las estrategias, los resultados son coherentes con lo hallado en la literatura internacional. Las estrategias fueron en general intensivas en el uso de recursos humanos. El 60\% de los costos del programa de vectores de Guantánamo, Cuba, correspondió a este recurso 19, de igual manera para el programa de control de vectores de Colombo, Sri Lanka, el recurso humano representó un $79 \%$ de los costos 20 y en Suramérica las rutinas de control de vectores en la ciudad de Salto, Uruguay, fueron intensivas en personal en un 60\% y en Machala, Ecuador, fue de un $66 \%$ del total de los costos 21 . Estos programas se caracterizaron por estar enfocados al control del vector adulto, criaderos de larvas y trabajo con la comunidad. No obstante, estrategias como la entrega de toldillos TILD son intensivas en el uso de capital, para Girón los resultados indicaron un 54\% del total de costos de la estrategia, esto concuerda con lo hallado para una intervención de entrega de toldillos TILD en Girardot, Colombia, donde el 78\% de los costos correspondió a capital (coste del toldillo, tratado como capital por tener una vida útil de 3 años) 21 .

A nivel de costo per cápita, el costo del programa de control de vectores en Girón fue de USD 0,88 y en Guadalajara de Buga fue de USD 0,99, estos valores contrastados con la experiencia internacional se encuentran por debajo de estudios como el de Guantánamo con un costo de USD 5,09 per cápita 19 o para las municipalidades del norte de Italia en la provincia de Emilia-Romagna con EUR 1,2 (USD 1,67 aproximadamente) per cápita 22. A nivel de costo por estrategias, Guantánamo registró un costo per cápita superior en la estrategia de control de vector adulto con un costo de USD 3,09 per cápita y USD 2 per cápita para el control larvario 19, mientras que en Girón el costo para esta estrategia fue de USD 0,23 per cápita y de USD 0,5 per cápita para Guadalajara de Buga y para la estrategia de control larvario fue de USD 0,05 per cápita para Girón y USD 0,4 per cápita para Guadalajara de Buga.

En cuanto a los costos por caso, los resultados hallados para Girón (USD 326 por caso) y Guadalajara de Buga (USD 117 por caso) resultan ser inferiores al reportado en las localidades de Malasia, donde se encuentra un costo de USD 499 por caso reportado 23. Desagregando por estrategias, el control de vectores adultos en las municipalidades de Malasia registraron un valor de USD 299 por caso 23 , mientras que los valores de costo por caso hallados para Girón fueron de USD 84 y para Guadalajara de Buga de USD 83 para la misma estrategia.

Vale la pena mencionar que este estudio presenta limitaciones, por una parte no se cuantificaron los bienes de capital, correspondientes a las edificaciones en las que funcionaban las dependencias de ETV, pues al no tener un bien comparador para valorar estos activos públicos, no fue incluido en el estudio. Este trabajo corresponde a un estudio de tipo descriptivo, por tanto, los resultados y comparaciones entre los municipios analizados no provienen de un análisis de inferencia estadística. Otra limitación que se puede destacar es que no se contrastó la ejecución de recursos con indicadores entomológicos recolectados por las localidades. Estos no se incluyeron, dado que no eran indicadores continuos que permitieran hacer un seguimiento, y no abarcaban la totalidad de las cabeceras urbanas de los municipios.

Finalmente, este estudio ilustra el costo de los programas de control de ETV en dos territorios de Colombia. La documentación de estos costos es relevante, dado que sirve como evidencia que conduzca a discutir sobre la mejora de la planificación de las estrategias de control de ETV, la administración de los recursos destinados al control y prevención de estas enfermedades, y como insumo para la realización de futuros estudios que se propongan evaluar la relación costo-efectividad de las estrategias de ETV en contextos locales. 


\section{Colaboradores}

M. A. Salinas-López contribuyó en la concepción de la idea de investigación, elaboración y revisión del manuscrito en todas sus versiones, aprobación de la versión final, procesamiento y análisis de los datos, responsabilidad de los resultados expuestos y en los análisis derivados de los datos. V. E. SotoRojas participó en la consecución de la documentación y análisis de los datos recolectados de costos de los programas de control vectorial, concepción y diseño de la investigación; en la revisión crítica y corrección de las versiones del artículo. C. B. Ocampo participó en la revisión crítica y corrección de las versiones del artículo, consecución de los datos, análisis de la información epidemiológica y asesoría en entomología.

\section{Agradecimientos}

Se agradece a la Secretaría de Salud Girón y su respectiva coordinación de enfermedades trasmitidas por vectores, a la Secretaría de Salud de Guadalajara Buga y su coordinación de medio ambiente, por el suministro de la información necesaria para realización de este estudio. A la Corporación CIDEIM, en especial a María Isabel Echavarría y Neila Julieth Mina por su orientación en el manejo de datos epidemiológicos y la literatura sobre las arbovirosis urbanas. Por último, se agradece al Centro de Estudios PROESA.

\section{Referencias}

1. Bhatt S, Gething PW, Brady OJ, Messina JP, Farlow AW, Moyes CL, et al. The global distribution and burden of dengue. Nature 2013; 496:504-7.

2. World Health Organization. Dengue: guidelines for diagnosis, treatment, prevention, and control. Geneva: Special Programme for Research and Training in Tropical Diseases/ World Health Organization; 2009.

3. Instituto Nacional de Salud. Boletín Epidemiológico Semanal 2016; (52).

4. Castrillón JC, Castaño J, Urcuqui S. Dengue en Colombia, diez años de evolución. Rev Chil Infectol 2015; 32:142-9.

5. Chaparro-Narváez P, León-Quevedo W, Castañeda-Orjuela CA. Comportamiento de la mortalidad por dengue en Colombia entre 1985 y 2012. Biomédica 2016; 36 Suppl 2:12534.

6. Shepard DS, Undurraga EA, Halasa YA, Stanaway JD. The global economic burden of dengue: a systematic analysis. Lancet Infect Dis 2016; 16:935-41.

7. Castro Rodríguez R, Carrasquilla G, Porras A, Galera-Gelvez K, Yescas JG, Rueda-Gallardo JA. The burden of dengue and the financial cost to Colombia, 2010-2012. Am J Trop Med Hyg 2016; 94:1065-72.

8. Departamento Administrativo Nacional de Estadística. Defunciones no fetales 2010. https:// www.dane.gov.co/index.php/estadisticaspor-tema/salud/nacimientos-y-defunciones/ defunciones-no-fetales/defunciones-no-fe tales-2010 (accedido el 09/Ago/2017).

9. Stanaway JD, Shepard DS, Undurraga EA, Halasa YA, Coffeng LE, Brady OJ, et al. The global burden of dengue: an analysis from the Global Burden of Disease Study 2013. Lancet Infect Dis 2016; 16:712-23.

10. Instituto Nacional de Salud. Protocolo para la vigilancia en salud pública del dengue. http://www.ins.gov.co/temas-de-interes/Den gue/01 Protocolo Dengue.pdf (accedido el 12/ $\mathrm{Jul} / 2017)$.

11. Castro R, Galera K, López JG, Rueda JA. Costs of dengue to the health system and individuals in Colombia from 2010 to 2012. Am J Trop Med Hyg 2015; 92:709-14.

12. Hendriks ME, Kundu P, Boers AC, Bolarinwa OA, te Pas MJ, Akande TM, et al. Step-by-step guideline for disease-specific costing studies in low- and middle-income countries: a mixed methodology. Glob Health Action 2014; 7:23573

13. Johns B, Baltussen R, Hutubessy R. Cost effectiveness and resource programme costs in the economic evaluation of health interventions. Cost Eff Resour Alloc 2003; 1:1.

14. Cafferata ML, Bardach A, Rey-Ares L, Alcaraz A, Cormick G, Gibbons L, et al. Dengue epidemiology and burden of disease in Latin America and the Caribbean: a systematic review of the literature and meta-analysis. Value Health Reg Issues 2013; 2:347-56. 
15. Erlanger TE, Enayati AA, Hemingway J, Mshinda H, Tami A, Lengeler C. Field issues related to effectiveness of insecticide-treated nets in Tanzania. Med Vet Entomol 2004; 18:153-60.

16. Departamento Nacional de Planeación. Fichas de caracterización territorial. https://colaboracion.dnp.gov.co/CDT/DesarrolloTerri torial/FIT/PDF/68307.pdf (accedido el 21/ $\mathrm{Jul} / 2017)$.

17. Departamento Nacional de Planeación. Fichas de caracterización territorial. https://colaboracion.dnp.gov.co/CDT/DesarrolloTerri torial/FIT/PDF/76111.pdf (accedido el 21/ $\mathrm{Jul} / 2017)$.

18. Departamento Nacional de Planeación. Guía orientaciones para realizar la medición de desempeño integral municipal. Guía para oficinas departamentales de planeación. https:// colaboracion.dnp.gov.co/CDT/DNP/EI-G01 Guía orientaciones para realizar la medición de desempeño municipal.Pu.pdf (accedido el 17/Ene/2018)

19. Baly A, Toledo ME, Lambert I, Benitez E, Rodriguez K, Rodriguez E, et al. Cost of intensive routine control and incremental cost of insecticide-treated curtain deployment in a setting with low Aedes aegypti infestation. Rev Soc Bras Med Trop 2016; 49:418-24.
20. Thalagala N, Tissera H, Palihawadana P, Amarasinghe A, Ambagahawita A, Wilder-Smith A, et al. Costs of dengue control activities and hospitalizations in the public health sector during an epidemic year in urban Sri Lanka. PLoS Negl Trop Dis 2016; 10:e0004466.

21. Alfonso-Sierra E, Basso C, Beltrán-Ayala E, Mitchell-Foster K, Quintero J, Cortés S, et al. Innovative dengue vector control interventions in Latin America: what do they cost? Pathog Glob Health 2016; 110:14-24.

22. Canali M, Rivas-Morales S, Beutels P, Venturelli C. The cost of arbovirus disease prevention in Europe: area-wide integrated control of tiger mosquito, Aedes albopictus, in EmiliaRomagna, Northern Italy. Int J Environ Res Public Health 2017; 14:444.

23. Packierisamy PR, Ng C-W, Dahlui M, Inbaraj J, Balan VK, Halasa YA, et al. Cost of dengue vector control activities in Malaysia. Am J Trop Med Hyg 2015; 93:1020-7. 


\section{Abstract}

The aim of this study was to measure the costs of vector-borne disease control programs at the local level in Colombia (2016). A cost analysis was performed for this purpose from the policymaker's perspective in the municipalities (counties) of Girón and Guadalajara de Buga, specifically for the Aedes aegypti control program, the principal mosquito vector of dengue, Zika, and chikungunya. The analysis involved the quantification of all the costs required for each of the prevention and control strategies in vector-borne diseases. The costs were classified as operating and capital costs, and for purposes of comparison the costs were also calculated per case and per capita. The programs' total estimated costs were USD 146,651 in Girón and USD 97,936 in Guadalajara de Buga. Per capita cost was USD 0.88 in Girón and USD 0.99 in Guadalajara de Buga. In general, the predominant cost strategies were chemical spraying of adult mosquitos, accounting for 26\% of the total costs in Girón and 47\% in Guadalajara de Buga, with personnel representing $40 \%$ of the total costs for this strategy in Giron and 66\% of the operating costs in Guadalajara de Buga.

Dengue; Costs and Cost Analysis; Vector Control

\section{Resumo}

O objetivo deste estudo foi quantificar os custos dos programas de controle de doenças transmitidas por vetores ao nível local na Colômbia em (2016). Para isso, foi realizada uma análise de custos desde a perspectiva do criador de politicas nos municípios de Girón e Guadalajara de Buga, especificamente para o programa de controle do Aedes aegypti, principal vector transmissor da dengue, Zika e chikungunya. Esta análise implicou a quantificação de todos os custos requeridos para cada uma das estratégias de prevenção e controle de doenças transmitidas por vetores. Os custos foram classificados em recorrentes e do capital, ademais, para fins comparativos foi obtido o custo por caso e nivel per capita. O custo total estimado dos programas foi de USD 146.651 no Município de Girón e USD 97.936 no Município de Guadalajara de Buga; ao nível per capita o custo foi de USD 0,88 em Girón e em Guadalajara de Buga foi de USD 0,99. Em geral, as estratégias predominantes em custos foram o controle químico de vetores adultos, com um 26\% do total dos custos em Girón e um 47\% em Guadalajara de Buga, essa foi intensiva em custos recorrentes, com um custo de pessoal de um 40\% do total de custos para esta estratégia em Girón e um 66\% de custos operacionais em Guadalajara de Buga.

Dengue; Custos e Análise de Custo; Controle de Vetores
Recibido el 06/Mar/2018

Versión final presentada el 19/Jul/2018

Aprobado el 17/Ago/2018 